\title{
Effect of Etching on the Optical, Morphological Properties of Ag Thin Films for SERS Active Substrates
}

\author{
Desapogu Rajesh, M. Mahendar, and C. S. Sunandana \\ School of Physics, University of Hyderabad, Hyderabad 500046, India \\ Correspondence should be addressed to C. S. Sunandana; sunandana@gmail.com
}

Received 6 May 2013; Accepted 19 June 2013

Academic Editor: Kevin Shuford

Copyright (C) 2013 Desapogu Rajesh et al. This is an open access article distributed under the Creative Commons Attribution License, which permits unrestricted use, distribution, and reproduction in any medium, provided the original work is properly cited.

\begin{abstract}
Structural, optical, and morphological properties of Ag thin films before and after etching were investigated by using X-ray diffraction, UV-Vis spectrophotometer, and field emission scanning electron microscopy (FESEM). The $\mathrm{HNO}_{3}$ roughened Ag thin films exhibit excellent enhancement features and better stability than pure Ag thin films. Further, the Ag nanostructures are covered with Rhodamine 6G (Rh6G) and then tested with surface enhanced raman spectroscopy (SERS) for active substrates. Etched Ag films were found to exhibit a strong SERS effect and excellent thermal stability. Hence, the present method is found to be useful in the development of plasmon-based analytical devices, especially SERS-based biosensors.
\end{abstract}

\section{Introduction}

Surface-enhanced Raman scattering (SERS) is a sensitive tool for exploring metal/adsorbate interactions and reactivity of adsorbed species [1-10]. Since Fleischmann et al's initial study [1], SERS has been subjected to extensive theoretical and experimental investigations. Surface plasmon resonance (SPR), which is associated with collective electron resonance induced by incident light on a rough metal surface, is one of the electromagnetic effects contributing largely to the SERS phenomenon. In addition to their easy preparation and chemical stability, this kind of monolayer can find practical applications such as those in the area of highly resistant surface modifications [11-14]. It also provides insight into the structure and molecular packing of monolayer and multilayer organic films that is now a growing area of material research [15]. Knowledge of structure is the key to understand the relationship between microscopic structure and the macroscopic physical and chemical properties. SERS is an important analytical tool which allows the study of molecules adsorbed on a nanostructured rough surface or nanoparticles of noble metals (Ag, $\mathrm{Cu}$, and $\mathrm{Au}$ ) [16, 17]. Development of the SERS technique provides the possibility to obtain Raman spectra from a very low amount of substance, even from a single molecule [18-22]. The SERS effect leads to a large enhancement factor relative to normal Raman scattering. The SERS effect relates to the localized surface plasmon resonance of $\mathrm{Ag}$ nanoparticles, which is manifested in the absorption spectrum as an intensive and broad band. The SERS active substrates for broad applications are of great need in the field of molecular detection and biosensor applications. Herein we aim to report a study of optical properties on the etching rate dependence of the surface-induced morphology of Ag thin films for SERS active substrates.

\section{Experimental Part}

The experimental procedure of thermal evaporated Ag thin films is explained in our previous studies [23]. The prepared Ag thin films having $60 \mathrm{~nm}$ thick and used as such for etching with diluted $\mathrm{HNO}_{3}\left(1: 10, \mathrm{HNO}_{3}\right.$ and distilled water) at room temperature for $20 \mathrm{~s}$. This procedure is similar to the etching method for formation mechanism of $\gamma$-AgI thin films by Sunandana and Senthil Kumar [24]. The pure, etched $\mathrm{Ag}$ films structural properties were studied by X-ray diffraction (XRD) and the optical absorption of Ag thin films was determined by JASCO optical spectrophotometer. The optical absorption was measured in the UV-Visible range $300 \mathrm{~nm}$ to $800 \mathrm{~nm}$. FESEM techniques were used to evaluate 


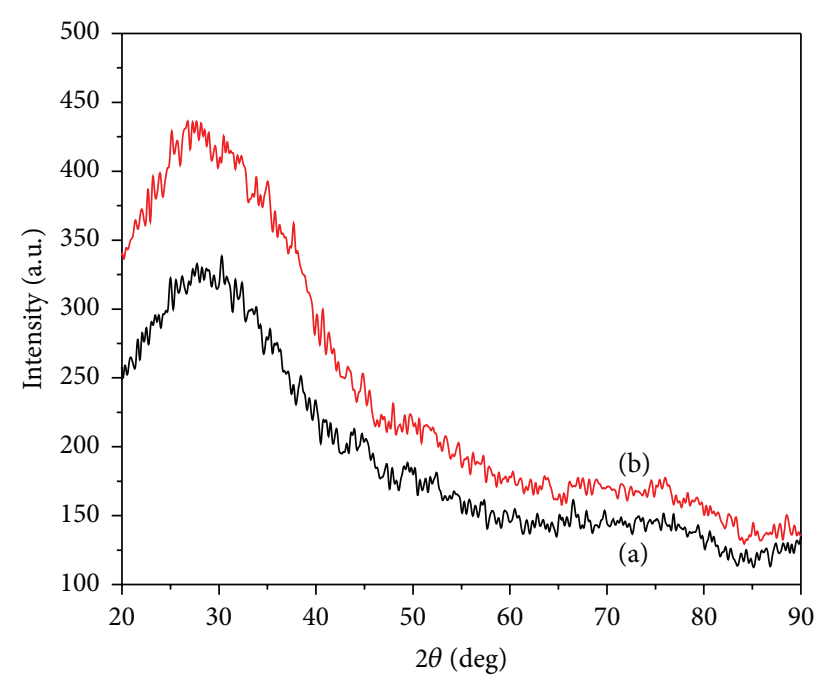

FIgURE 1: XRD pattern of $60 \mathrm{~nm}$ Ag film. (a) Pure, (b) $20 \mathrm{~s}$ etched Ag films.

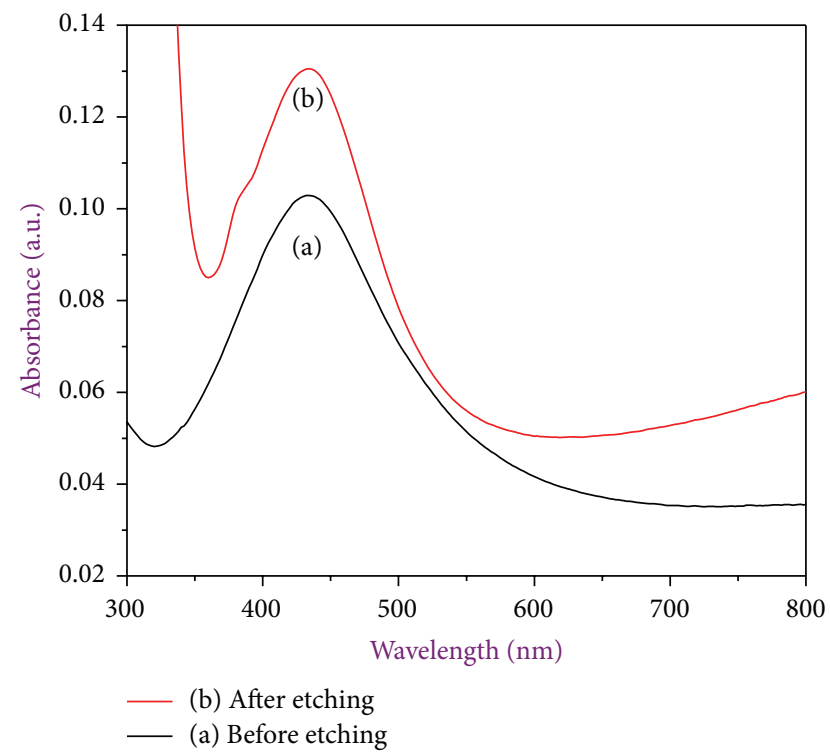

Figure 2: Absorbance spectrum of Ag films. (a) Pure Ag film (b) Ag film $20 \mathrm{~s}$ etched with diluted $\mathrm{HNO}_{3}$.

the microstructure elemental analysis of the samples and compared with the SPA 400 atomic force microscopic (AFM) results by using noncontact dynamic force mode (DFM). Finally SERS measurements were carried out by using microRaman (LABRAM-HR) studies with the 514.5 excitation wavelength and in the low concentration $\left(10^{-12} \mathrm{M}\right)$ of Rh6G.

\section{Results and Discussion}

3.1. Structure and Optical Properties. From X-ray diffraction (XRD) (see Figure 1), it was observed that the thermal evaporated $\mathrm{Ag}$ thin films have no crystalline phase, but only a broad huge peak observed at $30^{\circ}$ degrees. The $60 \mathrm{~nm} \mathrm{Ag}$ thin films XRD pattern shows a quasi-amorphous nature, and after etching these films show again a high intensity broader peak with large full width half-maxima (FWHM). These results indicate the increase in particle size and decrease in density of particles which are in agreement with FESEM/AFM results (see Section 3.2) and these results resemble the Raman enhancement.

Later, UV-VIS absorption spectra have been proved to be quite sensitive to the formation of silver, since Ag nanoparticles exhibit an intense absorption peak due to the surface plasmon excitation. Figure 2 shows that the intensity of SPR band and its full width at half-maxima (FWHM) increase with etching time. There was no obvious change in peak position for etching, except for the increase of absorbance. These signatures of absorption spectrum are in accordance with the increased size of $\mathrm{Ag}$ nanoparticles. It is obvious that with the progress of etching reaction more and more $\mathrm{Ag}^{+}$are reduced and this results in the increase in size of silver $(\mathrm{Ag})$ nanoparticles through diffusion controlled mechanism. This tremendous increment in absorption spectrum has useful applications as chemical sensors and detectors as well as some sensitive spectroscopic measurements. With the increasing size of Ag nanoparticles, multiple transitions $(\sim 380,430 \mathrm{~nm})$ of surface plasmon become more prominent and this is reflected on the increase in absorption intensity of etched Ag thin films. These broad SPR (FWHM) peaks mainly depend on the etching rate, thickness of the film, dielectric constant, and preparation of the sample. One major reason for SPR broadening is electron surface scattering, which may be enhanced for very small clusters. For smaller particles, electrons reach the surface faster and scatter quickly, losing the coherence of collective oscillation. In this case of thickness, etching rate is playing important role; based on this we can tune the optical, structural properties.

3.2. Morphology and Raman Studies. The morphology of Ag thin films was examined by FESEM. Figure 3(a) shows the FESEM image of as-deposited $\mathrm{Ag}$ thin film having uniform distribution with small nanoagglomerated particles. In the case of etching nanoagglomerations (see Figure 3(b)) were completely isolated which is basically from the surface diffusion of $\mathrm{Ag}$ atoms. The average $\mathrm{Ag}$ nanoparticles sizes of unetched and etched films are estimated to be $65 \pm 5$ and $100 \pm 10 \mathrm{~nm}$, respectively. The electron dispersive scattering (EDS) spectrum (Figures 3(c) and 3(d)) clearly showing the purity of the Ag nanoparticle formation and no other peaks was detected. In Figures 4(a) and 4(b) as-prepared Ag thin films of two- and three-dimensional micrographs of AFM images are showing the average particle size is $84 \mathrm{~nm}$ and RMS roughness value is $1.2 \mathrm{~nm}$. After $20 \mathrm{~s}$ etching Ag thin films (see Figures 4(c) and 4(d)) are showing the average particle size is $100 \mathrm{~nm}$ and corresponding RMS roughness is $4.3 \mathrm{~nm}$, after etching particle size and RMS values were increased and this result agreed with FESEM results. These roughened $\mathrm{Ag}$ thin films are good candidate to improve the SERS enhancement and useful for sensor applications.

On the other hand, SERS is a routine and powerful tool for the investigation and structural characterization of interfacial and thin film systems [25]. Because the surface 


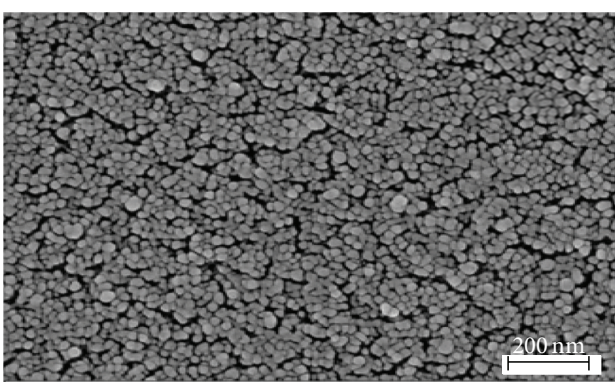

(a)

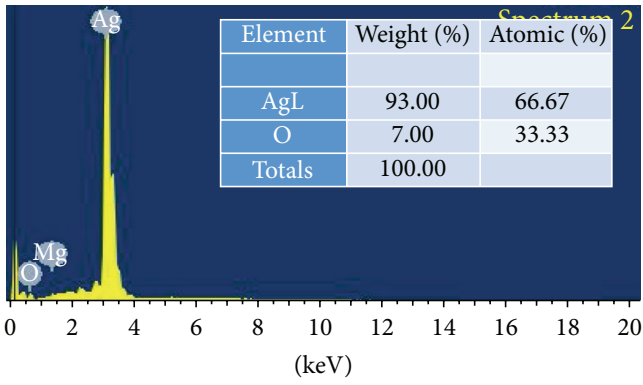

(c)

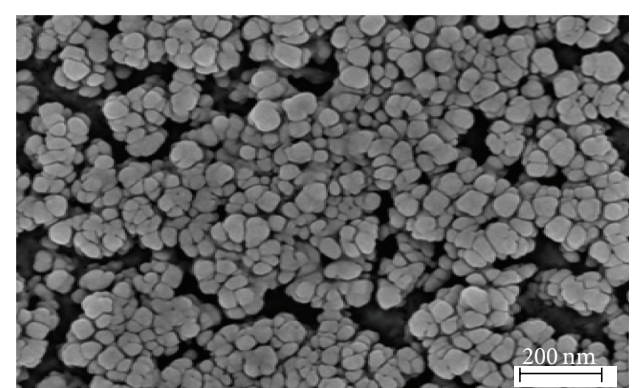

(b)

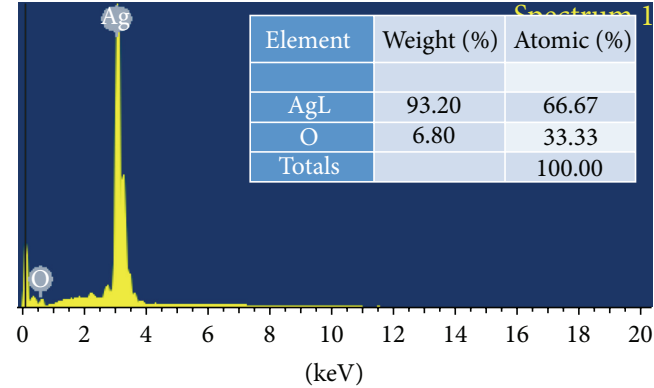

(d)

FIGURE 3: (a) FESEM images show complete agglomeration in $60 \mathrm{~nm}$ Ag thin film. (b) FESEM image of $60 \mathrm{~nm}$ Ag film etched for $20 \mathrm{~s}$ with diluted $\mathrm{HNO}_{3}$ in atmosphere, showing Iceland separation and agglomeration. (c) As-prepared Ag thin films EDS spectrum. (d) After etching EDS spectrum.

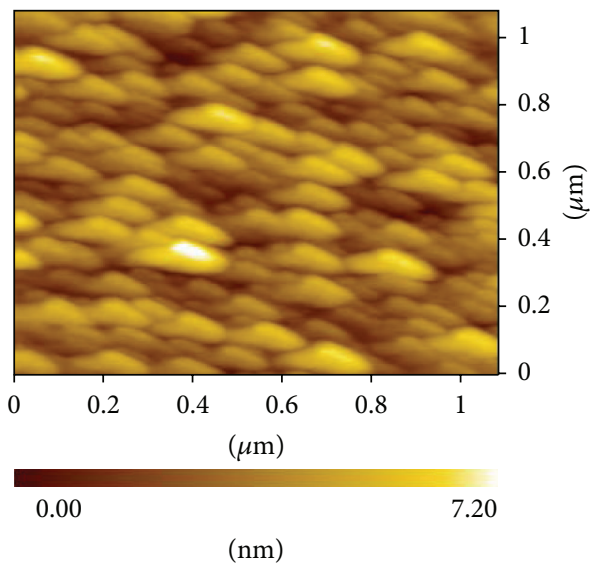

(a)

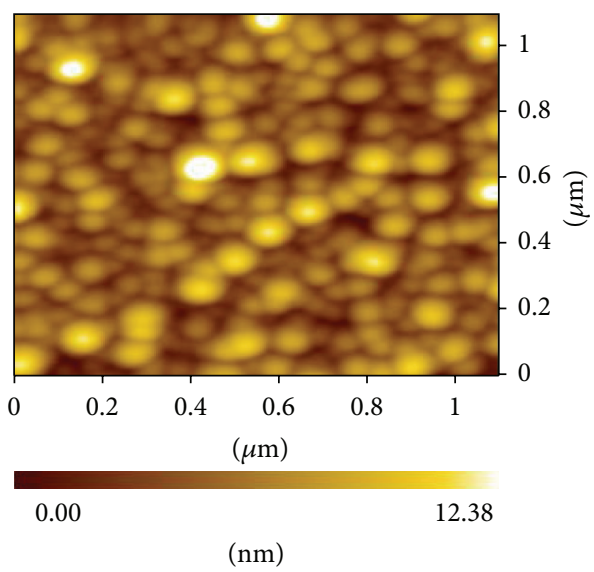

(c)

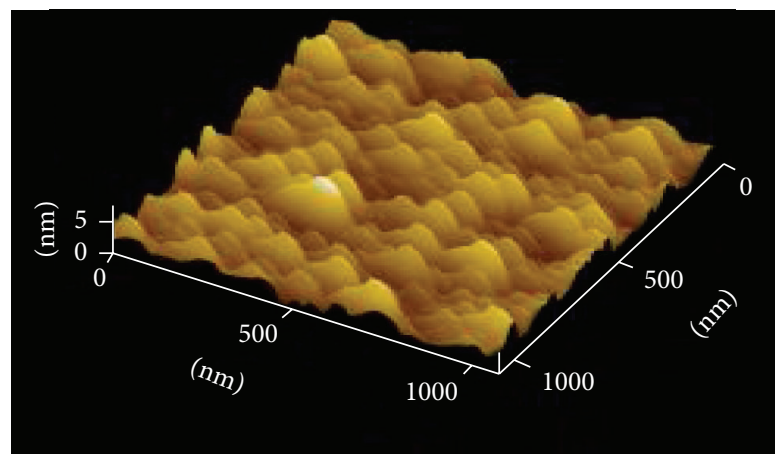

(b)

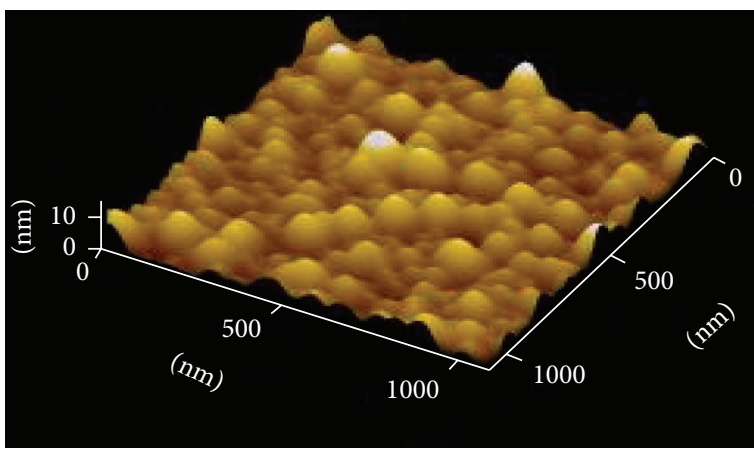

(d)

Figure 4: (a) and (b) Ag thin films of two-, three-dimensional atomic force microscopic pictures before etching. (c) and (d) Ag thin films of two-, three-dimensional atomic force microscopic pictures after etching with $\mathrm{HNO}_{3}$. 


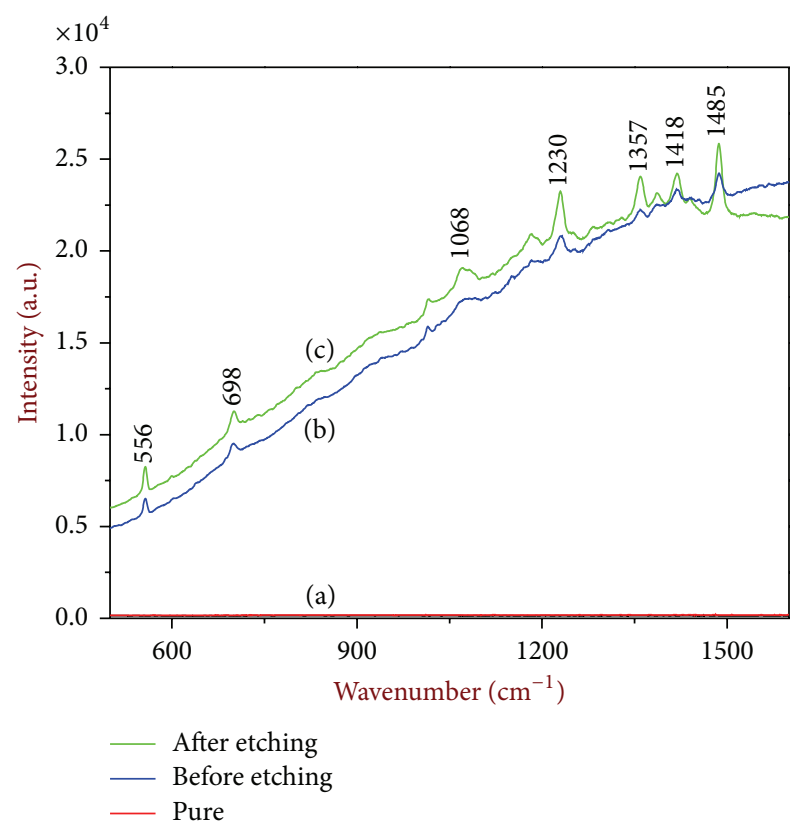

FIGURE 5: SERS spectrum of Rhodamine 6G absorbed on Ag film, (a) Rhodamine 6G $\left(3 \times 10^{-12} \mathrm{M}\right)$, (b) (Rhodamine $6 \mathrm{G}+\mathrm{Ag}$ films) before etching, and (c) (Rhodamine $6 \mathrm{G}+\mathrm{Ag}$ films) after etching.

morphologies and structures of the substrates determine the generation of intensified Raman signals, the substrates play a vital role in SERS and the research on the SERS active substrates remains a hot topic. An excellent SERS spectrum was obtained even at low concentration $\left(10^{-12} \mathrm{M}\right)$ of Rh6G as shown in Figure 5. The $\mathrm{HNO}_{3}$ roughened $\mathrm{Ag}$ thin films exhibit excellent stability for enhancement because the acid roughened $\mathrm{Ag}$ film features a strong enhancement factor better stability than pure Ag thin films. This preliminary result shows that a careful optimization of film thickness and etching time would lead to SERS quality films. In summary, we found that very stable and optically tunable SERS active Ag films can be reproducibly fabricated simply by etching method. Hence, the method will be useful in the development of plasmon-based analytical devices, specifically SERS-based biosensors [26].

\section{Conclusion}

The Ag thin films exhibit surface plasmon absorption characteristics. Based on the etching mechanism, the etching rate changes the morphology of the $\mathrm{Ag}$ thin films and it is confirmed by FESM. The $\mathrm{HNO}_{3}$ roughened Ag film seems to be a more routinely preparable substrate system for SERS study. The $\mathrm{HNO}_{3}$ roughened $\mathrm{Ag}$ thin films exhibit excellent stability for enhancement because the acid roughened $\mathrm{Ag}$ films feature a strong enhancement factor better stability than pure Ag thin films.

\section{Acknowledgment}

D. Rajesh thanks the School of Physics, University of Hyderabad, for providing good experimental facilities.

\section{References}

[1] M. Fleischmann, D. J. Hendra, and A. J. Mc Quillan, "Raman spectra of pyridine adsorbed at a silver electrode," Chemical Physics Letters, vol. 26, pp. 163-166, 1947.

[2] D. L. Jeanmaire and R. P. van Duyne, "Surface Raman spectroelectrochemistry Part I. Heterocyclic, aromatic, and aliphatic amines adsorbed on the anodized silver electrode," Journal of Electroanalytical Chemistry, vol. 84, no. 1, pp. 1-20, 1977.

[3] M. G. Albrecht and J. A. Creighton, "Anomalously intense Raman spectra of pyridine at a silver electrode," Journal of the American Chemical Society, vol. 99, no. 15, pp. 5215-5217, 1977.

[4] P. R. van Duyne, in Chemical and Biochemical Applications of Lasers, C. B. Moore, Ed., vol. 4, pp. 101-185, Academic Press, New York, NY, USA, 1979.

[5] T. E. Furtak and R. K. Chang, Eds., Surface Enhanced Raman Scattering, Plenum Press, New York, NY, USA, 1982.

[6] M. Moskovits, "Surface-enhanced spectroscopy," Reviews of Modern Physics, vol. 57, no. 3, pp. 783-826, 1985.

[7] A. Otto, I. Mrozek, H. Grabhorn, and W. Akemann, "Surfaceenhanced Raman scattering," Journal of Physics: Condensed Matter, vol. 4, no. 5, article, pp. 1143-1212, 1992.

[8] T. Vo-Dinh, "Surface-enhanced Raman spectroscopy," in Photonic Probes of Surfaces, P. Halevi, Ed., vol. 1, p. 67, Elsevier, Amsterdam, The Netherlands, 1995.

[9] T. M. Cotton, in Spectroscopy of Surface, R. J. H. Clark and R. E. Hester, Eds., p. 91, John Wiley \& Sons, Chichester, UK, 1993.

[10] A. Campion, "Surface-enhanced Raman scattering," Chemical Society Reviews, vol. 27, no. 4, pp. 241-250, 1998.

[11] D. Rajesh and C. S. Sunandana, "Controlled structural and optical properties of nanostructured Ag films reshaped by brief iodization," Thin Solid Films, vol. 524, pp. 316-319, 2012.

[12] D. Rajesh and C. S. Sunandana, "Briefly brominated Ag thin films: XRD, FESEM, and optical studies of surface modification," Applied Surface Science, vol. 259, pp. 276-282, 2012.

[13] D. Rajesh and C. S. Sunandana, "Effect of etching on the optical properties of partially iodized commercial silver foils," Indian Journal of Physics, vol. 86, no. 8, pp. 681-686, 2012.

[14] T. Dadosh, "Synthesis of uniform silver nanoparticles with a controllable size," Materials Letters, vol. 63, no. 26, pp. 22362238, 2009.

[15] J. D. Swalen, D. L. Allara, J. D. Andrade et al., "Molecular monolayers and films," Langmuir, vol. 3, no. 6, pp. 932-950, 1987.

[16] M. Moskovits, "Surface-enhanced Raman spectroscopy: a brief retrospective," Journal of Raman Spectroscopy, vol. 36, no. 6-7, pp. 485-496, 2005.

[17] K. Kneipp, H. Kneipp, I. Itzkan, R. R. Dasari, and M. S. Feld, "Surface-enhanced Raman scattering and biophysics," Journal of Physics Condensed Matter, vol. 14, no. 18, pp. R597-R624, 2002.

[18] N. Leopold and B. Lendl J, "A new method for fast preparation of highly surface-enhanced Raman scattering (SERS) active silver colloids at room temperature by reduction of silver nitrate with hydroxylamine hydrochloride," The Journal of Physical Chemistry B, vol. 107, no. 24, pp. 5723-5727, 2003.

[19] Y. Wang, H. Chen, S. Dong, and E. Wang, "Fabrication and characterization of SERS-active silver clusters on glassy carbon," Journal of Raman Spectroscopy, vol. 38, no. 5, pp. 515-521, 2007.

[20] K. Kneipp, Y. Wang, H. Kneipp et al., "Single molecule detection using surface-enhanced Raman scattering (SERS)," Physical Review Letters, vol. 78, no. 9, pp. 1667-1670, 1997. 
[21] K. Kneipp, H. Kneipp, V. Bhaskaran Kartha et al., "Detection and identification of a single DNA base molecule using surfaceenhanced Raman scattering (SERS)," Physical Review E, vol. 57, no. 6, pp. R6281-R6284, 1998.

[22] P. J. G. Goulet, N. P. W. Pieczonka, and R. F. Aroca, "Mapping single-molecule SERRS from Langmuir-Blodgett monolayers on nanostructured silver island films," Journal of Raman Spectroscopy, vol. 36, no. 6-7, pp. 574-580, 2005.

[23] D. Bharathi Mohan, A. Philip, and C. S. Sunandana, "Iodization of antimony thin films: XRD, SEM and optical studies of nanostructured SbI3," Vacuum, vol. 82, no. 6, pp. 561-565, 2008.

[24] P. S. Kumar, P. B. Dayal, and C. S. Sunandana, "On the formation mechanism of $\gamma$-AgI thin films," Thin Solid Films, vol. 357, no. 2, pp. 111-118, 1999.

[25] B. Sun, X. Jiang, S. Dai, and Z. Du, "Single-crystal silver nanowires: preparation and Surface-enhanced Raman Scattering (SERS) property," Materials Letters, vol. 63, no. 29, pp. 25702573, 2009.

[26] H. K. Park, J. K. Yoon, and K. Kim, "Novel fabrication of Ag thin film on glass for efficient surface-enhanced Raman scattering," Langmuir, vol. 22, no. 4, pp. 1626-1629, 2006. 

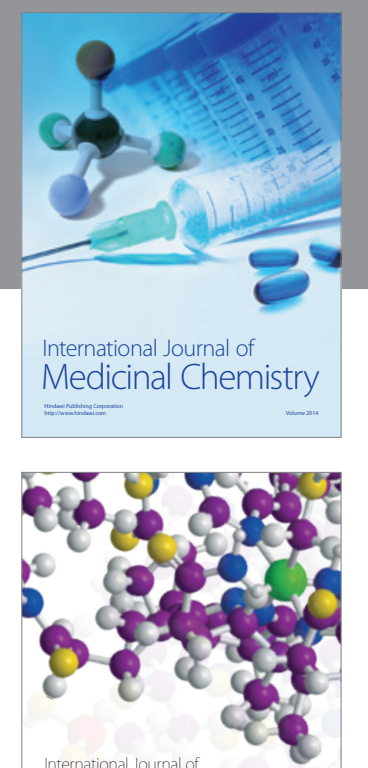

\section{Carbohydrate} Chemistry

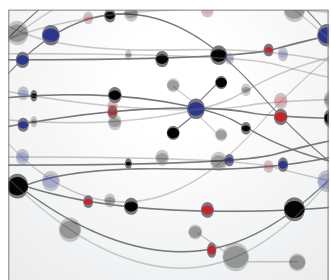

The Scientific World Journal
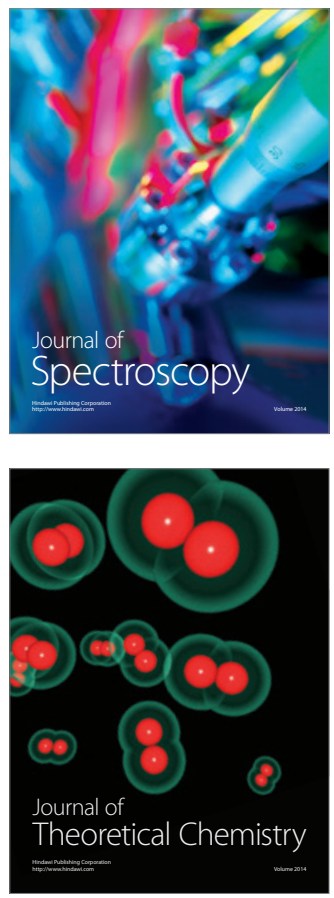
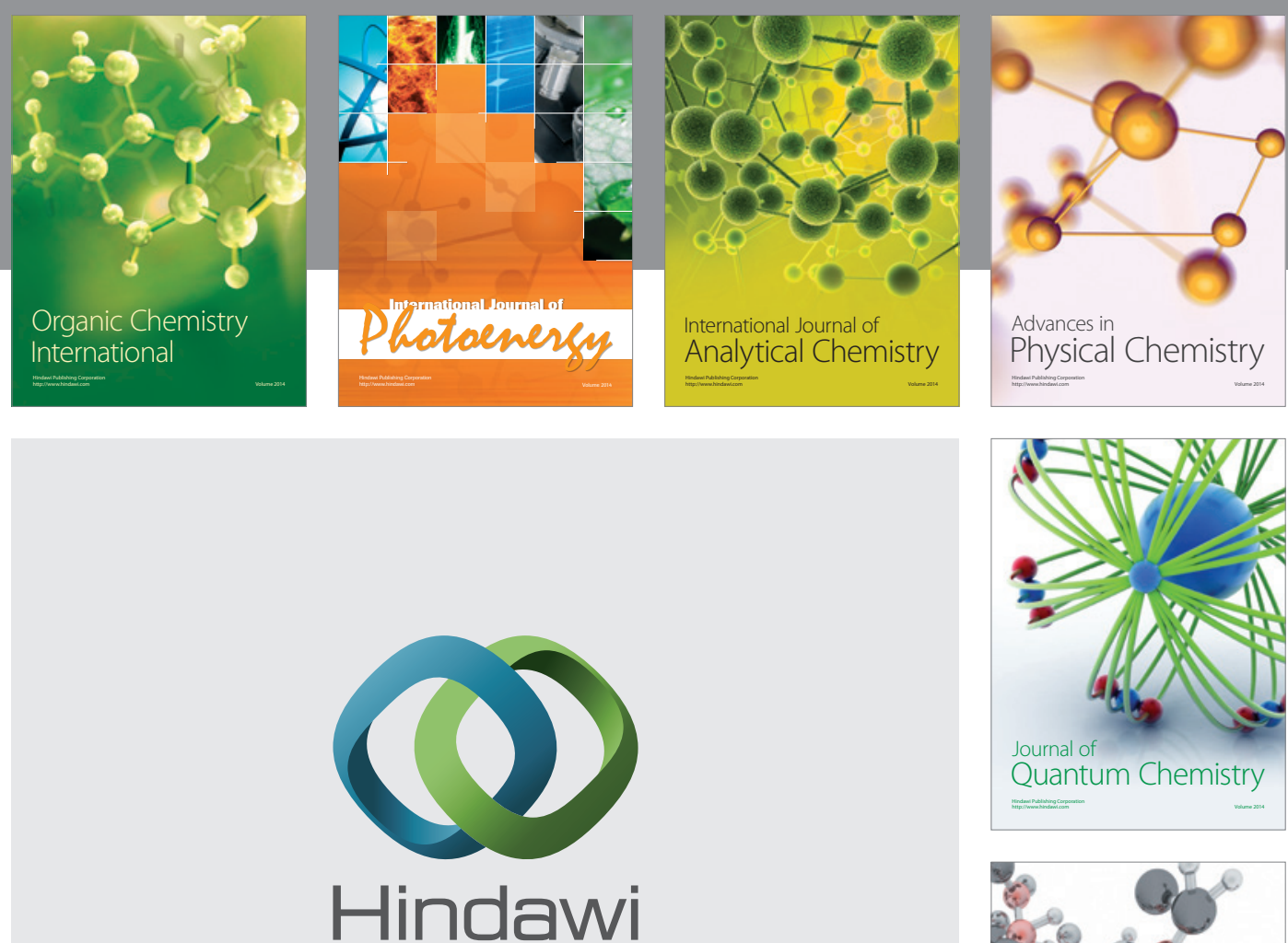

Submit your manuscripts at

http://www.hindawi.com

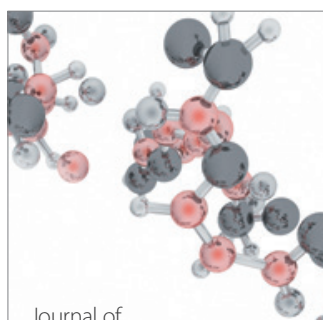

Analytical Methods

in Chemistry

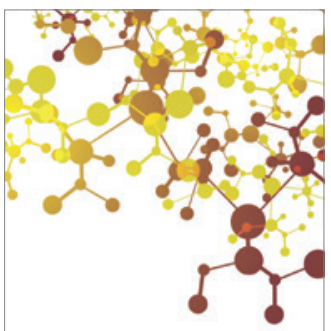

Journal of

Applied Chemistry

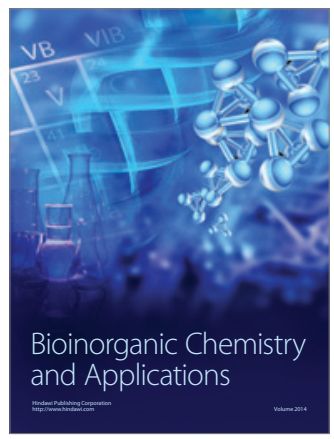

Inorganic Chemistry
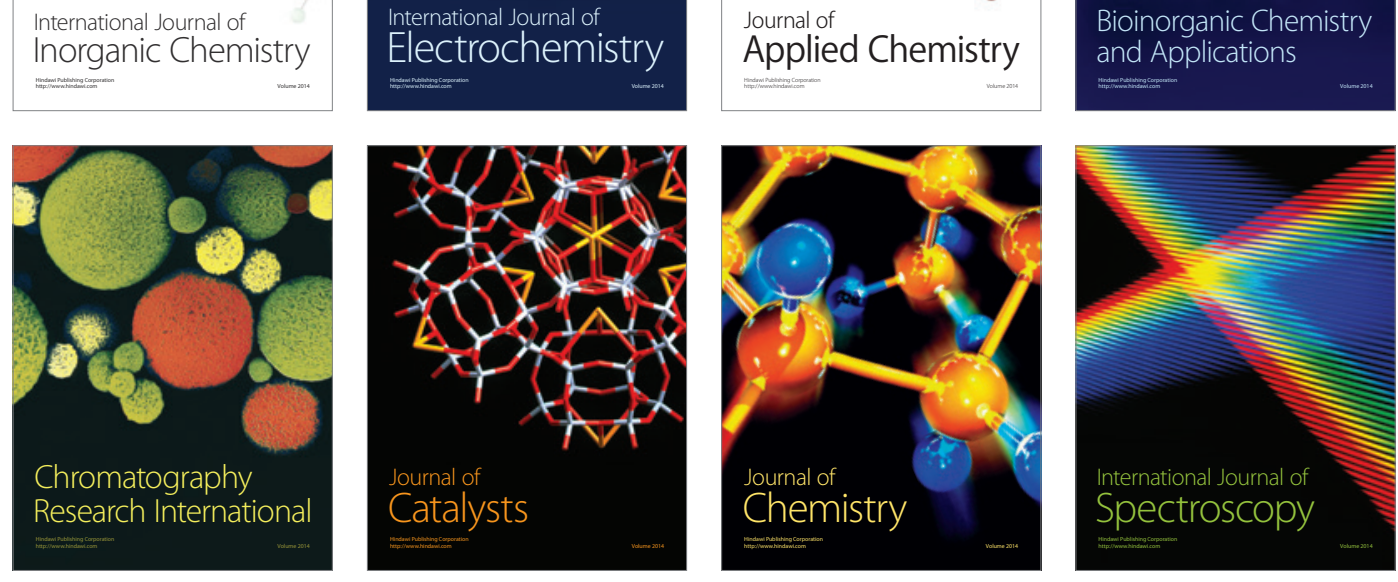